\title{
Virtual Reality as e-Mental Health to Support Starting with Mindfulness-Based Cognitive Therapy
}

\author{
Koen H.B. Damen ${ }^{1}$ and Erik D. van der Spek ${ }^{1}$ \\ ${ }^{1}$ Department of Industrial Design \\ Eindhoven University of Technology \\ Eindhoven, the Netherlands \\ k.h.b.damen@student.tue.nl, e.d.vanderspek@tue.nl* \\ *corresponding author
}

\begin{abstract}
Mindfulness-based cognitive therapy (MBCT) is used in mental health therapy but requires effort by the patient. This paper describes a preliminary design exploration of Open-MindEd, a Virtual Reality (VR) application specifically designed to support starting with MBCT. In collaboration with a mental health institute, two prospective users from the target group gave longitudinal input for the design, leading to the formulation of aspects how a VR application could serve as a tool to support people starting with mindfulness. Subsequently, a single-blind experiment $(\mathrm{N}=30)$ was performed to evaluate whether Open-MindEd could stimulate starting with MBCT. No significant positive effects were found. Despite several limitations, this paper shows some potential design considerations of VR as e-mental health to support MBCT.
\end{abstract}

Keywords: Virtual Reality; e-Mental Health; Mindfulness-Based Cognitive Therapy; Transtheoretical Model.

\section{Introduction}

According to the World Health Organization, around 20\% of adolescents in the world suffer from mental health disorders [18] with the most up to date inventory showing similar numbers for the Netherlands [7], where this study was performed. To combat rising costs, mental health organizations such as the GGZ (Dutch Association of Mental Health and Addiction Care), are exploring the possibilities of using e-mental health [6] for cognitive behavior therapy

Many e-mental health applications are based on cognitive behavior therapy [17]. A relatively novel form of cognitive behavior therapy is mindfulness-based cognitive therapy based on the work of Segal, Williams and Teasdale published in 2002 [16]. Mindfulness is "the awareness that emerges through paying attention on purpose, in the present moment, and nonjudgmentally to the unfolding of experience moment by moment" [9]. There are two types of mindfulness programs: mindfulness-based stress reduction (MBSR) [10] and mindfulness-based cognitive therapy (MBCT) [16]. At the GGZ, MBSR is an eight-week group program that uses mindfulness meditation to reduce suffering associated with (chronic) physical psychosomatic and psychiatric 
disorders [4]. MBCT is a manualized intervention based on the MBSR program. It incorporates elements of cognitive therapy that focus on detaching or decentering the view of one's thoughts. It is designed to prevent recidivating depression [1].

In practice, e-mental health for mindfulness is currently limited to online instructions for relaxation exercises and explanation of homework assignments [17]. However, homework assignments require effort by the patient, for cognitive therapy arguably even more so than stress reduction exercises, and failure by the patient to initiate this effort can thus harm the efficacy of the therapy. Therefore, new low-cost technologies are sought that can stimulate both starting with and sticking to therapy from home [17]. After a discussion with the GGZ, it was decided that Virtual Reality (VR) could be a possible avenue here, as it is relatively novel and can be used for persuasion [3].

\subsection{VR as a tool to stimulate uptake of mindfulness based cognitive therapy}

VR could be useful to stimulate self-management of MBCT from an ecological as well as a content perspective. First, it is hypothesized that people will be more compliant to start doing VR homework than currently due to the novelty effect of VR. While this would not lead to lasting change, for the setup of getting current patients to begin mindfulness training, we contend it is a viable effect. Subsequently, wearing a VR headset, the patient is unable to look away and so cannot get distracted by other stimuli.

Furthermore, a VR exercise system could increase intrinsic motivation for executing mindfulness exercises by 1 ) enabling users to "unlock” new exercises (stimulating competence [5]), and 2) providing the possibility to choose how long, how often and when to execute exercises, and which exercises to execute (stimulating autonomy [5]).

As we are primarily interested in improving the uptake of self-managed MBCT, the Transtheoretical Model (TTM) can be used to describe the moment that is most relevant for an intervention. The TTM lists stages of change to integrate processes and principles of various behavior change theories [14]. It consists of six stages of change: precontemplation, contemplation, preparation, action, maintenance, and termination. The steps between precontemplation and action best describe starting with a therapy.

Prochaska and Diclemente [13] found that the sample item "I tell myself I am able to quit smoking if I want to" is the best representor of self-liberation (making a firm commitment to change). Translating this to our research, "I tell myself I am able to start with mindfulness if I want to" best describes a commitment to behavior change. Therefore, the questionnaires used in the studies will stay close to this template.

\subsection{Related work}

A number of works have already examined VR for mindfulness, showing positive restorative results. VR mindfulness stress reduction exercises can reduce perceived pain for chronic patients [8], elicit deeper relaxation [11], reduce state anxiety in children [15], reduce urges of self-harm [12], and reduce the amount of complicated grief in grieving patients [2]. While evidence for the potential merit of VR stress reduction exercises for clinical settings is therefore rising, to the best of our knowledge the potential 
of VR to improve the uptake of mindfulness-based cognitive training, as well as its potential for self-management in home settings, has so far not been scrutinized.

\section{Open-MindEd}

In order to explore the design space and whether MBCT uptake for home users could be stimulated by VR in the context of therapy, Open-MindEd was developed. It consists of three different mindfulness attention exercises, co-designed with two participants currently undergoing MBCT and based on expert meetings with a therapist. In the codesign sessions, the participants among others said that they appreciated a feeling of safety in the environment while performing mindfulness exercises, and that VR could help with visualizing objects and environments. The exercises can be seen in Figure 1. One is a passive animation (no user interaction), where the patient had to focus on a sprout blooming into a flower. Users can set the time for the flower to bloom and thereby train shorter or longer-term attention. Another exercise is set in a forest and allows you to move in the direction that you are looking to (minimal interaction). It was designed to connect to the mindfulness experience of 'remembering to be', or being present in the environment. Lastly, a physics-based puzzle of placing an assortment of stones in the correct order on top of each other was developed (interaction with game mechanics); as this is a task that takes some effort to get right, it signified dealing with obstacles.

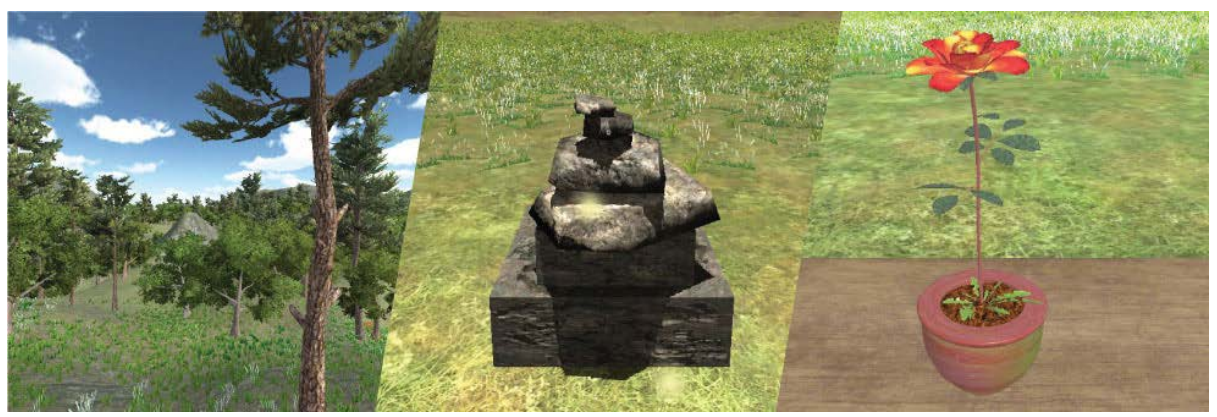

Fig. 1 - Three exercises: a walk through a forest (minimal interaction), placing stones on top of each other (interactive with game mechanics), watching a flower grow (passive).

\section{Case study on the design and perceived value}

With the help of a mindfulness trainer, a group of eight who just started with an eightweek MBCT-training (i.e. who were in the preparation stage of the TTM) were approached for a longitudinal case study. After being given the informed consent form a week in advance, two people volunteered; P1: F, 37, with persistent burn-out and anxiety feelings; P2: M, 24, mild social phobia. They were given a Galaxy S6 or S8 with Open-MindEd pre-installed, one Samsung Gear VR and a manual. Exercises were handed out in week 2 (nature walk), week 3 (stone stacking) and week 4 (focus on 
flower), with a subsequent semi-structured interview the week after each exercise. The interviews were audio recorded, transcribed and thematically analyzed according to the method of Zürn et al. [19]. The interviews gave some useful insights into the design. However, since only two persons participated they should be treated as anecdotal and in lieu of space constraints, we will only highlight some of the insights. A more detailed analysis is available upon request to the authors.

\section{Exercise 1: The Virtual Forest}

P1: "Because of that combination of the three sounds [wind, chirping birds, and water], you really think like: 'I am in the forest'." P2 said: "Immersion, the fact that you are really submerged into [the environment], also with your thoughts, that works really well." An advantage of this exercise is that "this makes it [mindfulness] quite tangible in my opinion" (P2). P1 said that "thanks to those goggles you are much more occupied with: 'What is happening now? What is happening at this moment?' It is almost literally that you are wearing blinkers."

\section{Exercise 2: Stacking Stones}

P1 found the touchpad controls frustrating, but regarded this as a good mindfulness exercise, as "you are quite focused on the here-and-now, because you are purely focusing on those stones". P2 really liked this exercise but he found it too easy as he was done quickly. That is why he suggested "when you have finished [stacking] it, that you immediately offer a new one."

\section{Exercise 3: The Virtual Flower}

The exercise was not really clear to P1. She thought that "it took quite long before something happened". However, when the leaves appeared on the stem "you suddenly get intrigued like: 'How is it going to look like?"' Therefore, P1 felt rewarded for waiting: "You are indeed rewarded for waiting for so long". P2 conversely found it difficult to focus: "my thoughts wandered off quite a lot". However, he managed to return his thoughts, "especially near the end when there was something to see". P1 believed that for "people who have real problems with executing breathing exercises, this might be a real good one". This exercise might also be very appropriate for people who have a lot of (ruminating) thoughts going on, according to P1. A disadvantage of this exercise would be that "it might be a visual meditation, but there might be not enough happening” (P2).

\section{General Remarks}

P1 liked that there were different exercises as it allows the user to try them all out and one can decide for him/herself what works best. Also, P1 liked "that you have something that you can grab and that centralizes you for a moment". However, she does "not know if I would immediately use VR goggles, because it also shuts you off quite drastically". According to $\mathrm{P} 2$, the reason "why this works really well with mindfulness, in my opinion, is because you can go to another environment in no-time. That is why [you can] leave your thoughts behind for an instance and return to them later." 
Both participants mentioned they would have liked to continue using the VR application for the training and, with some usability improvements, after as well.

\section{Experiment on the persuasive potential of VR mindfulness}

A small-scale experiment was performed to evaluate if Open-MindEd makes starting with executing mindfulness exercises more appealing for people without experience with mindfulness. As not enough people with similar mental health issues could be found for sufficient power, we chose to measure the effect on a general student population.

\section{Participants}

The participants were students from Eindhoven University of Technology (TU/e) with little to no experience with respect to mindfulness. $\mathrm{N}=30$ Participants were randomized evenly in an experiment group (10 female, mean age 21.3, SD 1.8), and a control group (7 female, mean age 21.1, SD 1.5). Familiarity with mindfulness and VR were not significantly different between the groups [resp. $\mathrm{F}(1,28)<1$ and $\mathrm{F}(1,28)=2.63, p=.12$ ]

\section{Procedure}

Students were incentivized by having a chance of winning 3x10 euros. After obtaining informed consent, participants were asked to rate the pre-intervention statements. Then the control group would execute a regular breathing exercise for ten minutes. The experiment group would execute the passive virtual flower exercise of Open-MindEd (see section 2) for ten minutes using a Samsung Gear VR with a Samsung Galaxy S6. This exercise was chosen because it did not introduce interactivity as an additional variable, therefore only comparing the VR modality of the exercise to that of a control group.

\section{Measurements}

Participants were asked to rate eight statements pre-intervention and twelve statements post-intervention about their ability and desire to start with mindfulness. Post-intervention, we also queried their opinion on the use of VR for mindfulness exercises. All statements were 7-point Likert scale items, with 1 being "strongly disagree", 4 being "neutral" and 7 being "strongly agree".

\subsection{Results}

Table 1 shows the pre- and post-intervention statement scores. ANCOVAs with the preintervention scores as covariates show no significant effect of the condition on postintervention scores [I would like to start with mindfulness: $\mathrm{F}(1,27)<1$; I am open to execute mindfulness exercises: $\mathrm{F}(1,27)<1$; I am able to start with mindfulness: $\mathrm{F}(1,27)$ $=1.22, p=.28$.

There was also no effect of the condition on how difficult the students found it to keep their thoughts focused $\mathrm{F}(1,28)=3.45, p=.07$, or how boring they found the exercises $\mathrm{F}(1,28)=1.76, p=.20$. We also measured subjective stress levels. All stress 
levels dropped but here too an ANCOVA with prior stress as covariate showed no significant effect of condition on post intervention stress levels $F(1,27)<1$.

Table 1. Pre- and post-intervention statement scores.

\begin{tabular}{|l|c|c|c|c|}
\hline \multicolumn{1}{|c|}{ Statement } & \multicolumn{2}{|c|}{ Control Group (M, SD) } & \multicolumn{2}{c|}{ VR Group (M, SD) } \\
\hline & Pre & Post & Pre & Post \\
\hline $\begin{array}{l}\text { I would like to start } \\
\text { with mindfulness }\end{array}$ & $4.50(1.38)$ & $4.65(1.44)$ & $4.93(0.94)$ & $5.10(1.04)$ \\
\hline $\begin{array}{l}\text { I am open to exe- } \\
\text { cute mindfulness } \\
\text { meditation exer- } \\
\text { cises }\end{array}$ & $5.63(0.97)$ & $5.73(.80)$ & $5.73(0.86)$ & $5.47(.92)$ \\
\hline $\begin{array}{l}\text { I am able to start } \\
\text { with mindfulness if } \\
\text { I want to }\end{array}$ & $5.25(1.56)$ & $5.83(1.08)$ & $5.10(1.24)$ & $5.47(.72)$ \\
\hline
\end{tabular}

\section{Conclusion}

From the experiment, no proof was found that the VR exercises persuaded participants to go from the precontemplation to the contemplation phase of MBCT exercises. Therefore, any novelty effect from the VR modality to motivate picking up mindfulness exercises should be considered negligible. If VR would make focusing thoughts easier and less boring, we also could not find evidence for that in our 10-minute experience. At the same time, it should be noted that the Flower focusing exercise used in the experiment was a passive VR experience, did not have competence enhancing game elements, nor did the experiment provide autonomy to choose exercises.

The case study with people that were already in the preparation phase conversely did show some interesting insights on how VR could be used to improve MBCT exercises for the target group. People could be supported to start with Mindfulness training by:

1. Shutting off users from their direct environment with the VR goggles, allowing them to focus on the here-and-now more easily;

2. Making mindfulness more tangible by providing clear assignments and visual representations;

3. Making it possible to go "somewhere else" in an instant and focus on something completely different, enabling users to break (negative) thought cycles;

4. Introducing exercises one at a time, while teasing users that more are coming soon;

5. Incorporating multiple exercises, without forcing users to execute an exercise more than once, so that users are able to pick the exercises that they like doing and work for them;

Due to the case study only having two participants, these points should however be considered anecdotal for now, and still require future testing. 


\section{References}

1. Baer, R. A. (2003). Mindfulness Training as a Clinical Intervention: A Conceptual and Empirical Review. Clinical Psychology: Science and Practice, 10(2), 125-143.

2. Botella, C., Osma, J., Palacios, A. G., Guillen, V., \& Banos, R.: Treatment of Complicated Grief using Virtual Reality: A Case Report. Death Studies, 32(7), 674-692 (2008).

3. Chittaro, L., \& Zangrando, N.: The Persuasive Power of Virtual Reality: Effects of Simulated Human Distress on Attitudes towards Fire Safety. In: International Conference on Persuasive Technology (pp. 58-69). Springer, Berlin, Heidelberg (2010).

4. Fjorback, L. O., Arendt, M., Ørnbøl, E., Fink, P., \& Walach, H.: MindfulnessBased Stress Reduction and Mindfulness-Based Cognitive Therapy - a systematic review of randomized controlled trials. Acta Psychiatrica Scandinavica, 124(2), 102-119 (2011).

5. Gagné, M., \& Deci, E. L.: Self-Determination Theory and Work Motivation. Journal of Organizational behavior, 26(4), 331-362 (2005).

6. GGZ Nederland website: E-mental health in the Netherlands. http://www.ggznederland.nl/uploads/assets/20130514\%20Factsheet\%20eHealth.pdf. Last accessed March 17, 2017.

7. De Graaf, R., Ten Have, M., Van Gool, C., \& Van Dorsselaer, S.: Prevalentie van psychische aandoeningen en trends van 1996 tot 2009; resultaten van NEMESIS2. Tijdschrift voor Psychiatrie, 54(1), 27 (2012).

8. Gromala, D., Tong, X., Choo, A., Karamnejad, M., \& Shaw, C. D.: The virtual meditative walk: virtual reality therapy for chronic pain management. In: Proceedings of the 33rd Annual ACM Conference on Human Factors in Computing Systems (pp. 521-524). ACM (2015).

9. Kabat-Zinn, J.: Mindfulness-Based Interventions in Context: Past, Present, and Future. Clinical psychology: Science and practice, 10(2), 144-156 (2003).

10. Kabat-Zinn, J. Full Catastrophe Living: Using the Wisdom of the Body and the Mind to Face Stress, Pain and Illness. New York, US: Dell (1990).

11. Kosunen, I., Salminen, M., Järvelä, S., Ruonala, A., Ravaja, N., \& Jacucci, G.: RelaWorld: neuroadaptive and immersive virtual reality meditation system. In: Proceedings of the 21st International Conference on Intelligent User Interfaces (pp. 208-217). ACM (2016).

12. Nararro-Haro, M. V., Hoffman, H. G., Garcia-Palacios, A., Sampaio, M., Alhalabi, W., Hall, K., \& Linehan, M.: The use of virtual reality to facilitate mindfulness skills training in dialectical behavioral therapy for borderline personality disorder: a case study. Frontiers in psychology, 7 (2016).

13. Prochaska, J. O., \& DiClemente, C. C.: Toward a comprehensive model of change. In Treating addictive behaviors (pp. 3-27). Springer US (1986). 
14. Prochaska, J. O., Redding, C. A., \& Evers, K. E.: The Transtheoretical Model and Stages of Change. In Health Behavior and Health Education: Theory, Research, and Practice ( $4^{\text {th }}$ ed.) (pp. 97-122). San Francisco, CA, US: John Wiley \& Sons, Inc (2008)

15. Van Rooij, M., Lobel, A., Harris, O., Smit, N., \& Granic, I.: DEEP: A biofeedback virtual reality game for children at-risk for anxiety. In: Proceedings of the 2016 CHI Conference Extended Abstracts on Human Factors in Computing Systems (pp. 1989-1997). ACM (2016).

16. Segal, Z.V., Williams, J.M., \& Teasdale, J.D.: Mindfulness-based cognitive therapy for depression: a new approach to preventing relapse. US: Guilford Press (2002).

17. Smeets, O.: E-mental health. Bijblijven, 32(5), 359-363 (2016).

18. World Health Organization website: http://www.who.int/features/factfiles/mental_health/mental_health_facts/en/Last accessed May 20, 2018.

19. Zürn, X., Damen, K., van Leiden, F., Broekhuijsen, M., \& Markopoulos, P.: Photo Curation Practices on Smartphones. In: 14th International Conference on Advances in Computer Entertainment Technology. Springer (2017). 